Check for updates

Cite this: RSC Adv., 2019, 9, 38982

\title{
Cd(II) removal by Fe(II) surface chemically modified layered double hydroxide-graphene oxide: performance and mechanism $\dagger$
}

\author{
Wei Liao, He Wang, Hui-qiang Li (D)* and Ping Yang
}

Cd(॥) adsorption onto Fe(॥) modified Layered double hydroxide-graphene oxide (LDH-GO@Fe(॥)) was investigated using batch experiments. With the modification of $\mathrm{Fe}(॥), \mathrm{LDH}-\mathrm{GO}$ maintained its structure, while Fe(॥) species formed non-crystalline iron oxide clusters on the surface of the LDH/GO. A kinetics study indicated that adsorption obeyed a pseudo-second-order rate law. The equilibrium data were fitted well with the Langmuir isotherm model. The maximum adsorption capacity of LDH-GO@Fe ()$_{10}$ was $28.98 \mathrm{mg} \mathrm{g}^{-1}$, higher those that of pure LDH-GO and LDH-GO@Fe $(\|)_{50}$. The increased sorption capacities could be explained by the increased specific surface area. Modification with Fe(॥) would lead to the generation of amorphous Fe oxides and Fe could occupy the binding sites for Cd(॥), thus excess Fe in the structure will restrain the adsorption of $\mathrm{Cd}\left({ }^{\prime \prime}\right)$. The XRD and XPS patterns revealed the formation of $\mathrm{Cd}(\mathrm{OH})_{2}$ after adsorption. Batch experiments indicated that precipitation and surface complexation were the main pathways for $\mathrm{Cd}(\Perp)$ removal.

Received 11th September 2019 Accepted 20th November 2019

DOI: 10.1039/c9ra07305a

rsc.li/rsc-advances

clay to remove $\mathrm{Cu}$ (II), which presented the $\mathrm{Cu}(\mathrm{II})$ maximum

\section{Introduction}

With the rapid development and extensive applications of heavy metals and their composites, release of heavy metals into the environment is inevitable and harmful. Cadmium is an extremely toxic heavy metal to humans and the environment even at low levels. ${ }^{1}$ The development of methods to remove trace potentially toxic metal contaminants from aqueous wastewater is one of the most important environmental issues being investigated. Among the techniques, the precipitation of metals as insoluble metal hydroxides via increment of the $\mathrm{pH}$ is the most applied approach. ${ }^{2}$ However, precipitation produces a large amount of sludge, and generates secondary pollution, and is incapable of removing trace metal contaminants. ${ }^{3,4}$ In comparison, adsorption has shown superiority in the removal of heavy metals and is extensively researched due to its low cost, high efficiency and easy operation. ${ }^{5}$ Different materials have been studied intensively to eliminate heavy metals from aqueous solutions. Most materials suffer from low efficiency or low adsorption capacity, which restrict their applications. ${ }^{6}$ Fiol et al. ${ }^{7}$ prepared $\mathrm{AC}$ from olive stone waste to remove heavy metals from aqueous solutions, and the highest value of the Langmuir maximum adsorption capacity for $\mathrm{Cd}(\mathrm{II})$ was found to be $7.73 \mathrm{mg} \mathrm{g}^{-1}$. Bertagnolli et al. ${ }^{8}$ employed a calcined bentonite

College of Architecture and Environment, Sichuan University, Chengdu 610065, China. E-mail: Lwei0314@163.com; wh_820@163.com; lhq_scu@163.com; yping63@163. com; Tel: +86 18200288011; +86 18011446490; +8618980668020; +8618602804508 $\uparrow$ Electronic supplementary information (ESI) available. See DOI: 10.1039/c9ra07305a adsorption capacity of $11.89 \mathrm{mg} \mathrm{g}^{-1}$.

In recent years, exploring hierarchical nanocomposites with multifunctional properties through combining various building blocks together into a well-designed structure has been a hot topic for material science. ${ }^{9}$ The composite materials composed of blocks with different physical and chemical properties can usually inherit full advantages of the component materials and even form multifunctional materials with unexpected properties for unique applications. ${ }^{10}$ Recent studies have documented that hierarchical composites have superb removal capacities for pollutants on the basis of the boosting of abundant active sites and favorable mass transfer, overcoming the demerits associated with agglomeration and low efficiency in the common nanoparticles. ${ }^{11}$ Over past few years, there are great interests in the synthesis of porous material supported layered double hydroxides (LDH) because of their high surface area, gap structure and unique property. ${ }^{12}$ The composites can provide stable sites for pristine LDH particles loading to restrain the aggregation of the particles to some extent, which thereby increases the surface area and stable sites for the removal of pollutants from environment. Among these composites, graphene materials supported LDH attracts the most research interests due to the superior physicochemical properties. With the combination, the disadvantage of aggregation can be effectively prevented, and their distinguishing properties can be integrated together. Graphene can provide the good electrical conductivity and high mechanical strength, while LDHs can provide good chemical reactivity. ${ }^{9}$ Thus, an electron pathway can be easily formed and a network of stress transfer can be also 
presented between the graphene and LDHs, which is an important aspect as advanced functional materials. In another aspect, such structured composites are always with a porous structure, which make the heat and mass transfers during a reaction greatly improved and make active sites easily exposed. ${ }^{10}$ Some studies on $\mathrm{LDH}$-graphene composites and their use for elimination of metal ions have been reported: $\mathrm{Pb}(\mathrm{II}),{ }^{13} \mathrm{U}(\mathrm{VI}){ }^{14}$ and $\mathrm{Cr}(\mathrm{VI}) .{ }^{15}$ Our study also showed the LDH-GO composites had an outstanding removal capacity for $\mathrm{Fe}(\mathrm{II}){ }^{\mathbf{1 6}} \mathrm{At}$ the $\mathrm{Fe}(\mathrm{II})$ concentration of $100 \mathrm{mg} \mathrm{L}^{-1}, \mathrm{Fe}_{2} \mathrm{O}_{3}$ and $\mathrm{FeOOH}$ were formed in the inner space of the $\mathrm{LDH}-\mathrm{G}$ and the structure of LDH-G had an obvious alteration. Introducing Fe has been reported to be one of the efficient methods for adsorbent modification. ${ }^{17-19}$ Iron-modified materials showed high affinity for metals due to their large surface area, microporous structure and internal surface. ${ }^{20}$ Doula $^{21}$ synthesized clin-Fe by adding natural clinoptilolite in an iron nitrate solution under strongly basic condition, and the clin-Fe was proven for its ability to adsorb high concentration $\mathrm{Cu}(\mathrm{II})$. Yang et al. ${ }^{22}$ reported corn stalk-derived biochar impregnate with iron nanoparticles to serve as an adsorbent, which showed rapid removal and high performance in single and mixed metal solutions. To the best of our knowledge, no attempt has been made to remove $\mathrm{Cd}$ (II) by Fe-decorated LDH-graphene composites. Fe-decorated LDHgraphene composites probably provide us a simple method for the efficient elimination of environmental pollutants from aqueous solution.

Therefore, the objectives of this study were (i) to characterize the Fe(II)-modified LDH/GO (LDH-GO@Fe(II)); (ii) to evaluate its adsorption kinetics and adsorption capacity for $\mathrm{Cd}(\mathrm{II})$; (iii) to elucidate the mechanisms involved in adsorption of Cd(II) by LDH-GO@Fe(II). Scanning electron microscopy, X-ray photoelectron spectroscopy, and X-ray powder diffraction patterns were used to better understand the mechanisms of $\mathrm{Cd}(\mathrm{II})$ removal. It is expected that the outcomes from the study would provide understanding for the application of $\mathrm{LDH}-\mathrm{GO} @ \mathrm{Fe}(\mathrm{II})$ as an efficient adsorbent for heavy metal removal.

\section{Materials and methods}

\subsection{Materials}

Purified natural graphite powder was purchased from Sinopharm Chemical Reagent Co. Ltd. Other reagents were analytical grade and purchased from Sigma Aldrich (Shanghai) Co. Ltd. without further purification. Glassware was soaked in 10$20 \% \mathrm{HCl}$ solution for $24 \mathrm{~h}$ and rinsed sequentially three times with distilled water.

\subsection{Fabrication of the adsorbents}

GO was synthesized by Hummers methods as described elsewhere. ${ }^{23}$ The detailed processes were illustrated in the ESI. $\dagger$ LDH-GO composites were synthesized via the co-precipitation methods. Briefly, $0.1 \mathrm{~g}$ of GO powder in $100 \mathrm{~mL}$ water was ultra-sonicated for $1 \mathrm{~h}$ and then stirred vigorously with a magnetic stirrer. A $100 \mathrm{~mL}$ mixed metal solution of $\mathrm{MgCl}_{2}$ $\cdot 6 \mathrm{H}_{2} \mathrm{O}$ and $\mathrm{AlCl}_{3} \cdot 9 \mathrm{H}_{2} \mathrm{O}$ was prepared in deionized water with a total metal ion concentration of $0.4 \mathrm{~mol} \mathrm{~L}^{-1}$ and a $\mathrm{Mg}^{2+} / \mathrm{Al}^{3+}$ molar ratio of $3: 1$. Another $100 \mathrm{~mL}$ alkaline solution of $0.6 \mathrm{~mol} \mathrm{~L}{ }^{-1} \mathrm{NaOH}$ and $0.2 \mathrm{~mol} \mathrm{~L}{ }^{-1} \mathrm{Na}_{2} \mathrm{CO}_{3}$ was also prepared. Both the mixed metal solution and alkaline solution were simultaneously dropwise added to the GO suspension under vigorous stirring at room temperature. The $\mathrm{pH}$ was maintained at $10 \pm 0.5$ during the process. The results suspension was stirred for another $4 \mathrm{~h}$ at room temperature, and then was aged in a water bath at $65^{\circ} \mathrm{C}$ for $4 \mathrm{~h}$. The precipitate was centrifuged, washed thoroughly with distill water until the washings were neutral. The precipitate was then dried at $65{ }^{\circ} \mathrm{C}$ overnight. The resulting powder was designated as $\mathrm{LDH}-\mathrm{GO}$. Hierarchical Fedecorated LDH-GO composites were prepared by adding a certain amount of LDH-GO to Fe(II)-containing $(5,10,50$, $100 \mathrm{mg} \mathrm{L}^{-1}$ ) solution and reacting for $1 \mathrm{~h}$, and noted as LDH$\mathrm{GO} @ \mathrm{Fe}(\mathrm{II})_{x}(x=5,10,50,100)$.

\subsection{Batch experiment}

The work Cd(II) solutions were prepared at various concentrations by diluting the stock Cd(II) solution with fresh distilled water. The experiments were carried out in a $250 \mathrm{~mL}$ glass conical flask by adding $0.2 \mathrm{~g}$ of adsorbents in $200 \mathrm{~mL}$ of the Cd(II)-containing solution with continuous stirring over a water bath magnetic stirrer at $500 \mathrm{rpm}$. If not otherwise specified, the solution $\mathrm{pH}$, temperature and initial concentration of cadmium in the cadmium solutions were $5,25{ }^{\circ} \mathrm{C}$ and $20 \mathrm{mg} \mathrm{L}^{-1}$, respectively. For kinetics study, the suspensions were withdrawn at a given specific time intervals and then immediately filtrated through a $0.45 \mu \mathrm{m}$ filter membrane, and the concentration of the residual cadmium in the filtrates was analyzed. Cadmium concentration was measured by the spectrophotometric method using 1-(2-pyrdylazo)-2-naphthol (PAN), ${ }^{24}$ the detailed operation is illustrated in the ESI. $\dagger$ Adsorbed cadmium was calculated from the difference between the initial cadmium concentration and the concentration that remained in the supernatant solution. Each experiment was conducted in duplicate. The dissolution of iron ions was monitored. No iron was detected during the process of $\mathrm{Cd}(\mathrm{II})$ sorption by $\mathrm{LDH}-$ GO@Fe(II) $)_{x}$. The blank checks were performed to verify the absence of adsorbate precipitation and/or adsorption to the walls of the vessels.

\subsection{Kinetic studies}

The pseudo-first-order and pseudo-second-order kinetic were used to analyze adsorption kinetic data. The linear form of the pseudo-first-order (1) and the pseudo-second-order (2) equations can be expressed as follows: ${ }^{25}$

$$
\begin{gathered}
\ln \left(q_{\mathrm{e}}-q_{t}\right)=\ln q_{\mathrm{e}}-k_{1} t \\
\frac{1}{q_{t}}=\frac{1}{k_{2} t q_{\mathrm{e}}^{2}}+\frac{1}{q_{\mathrm{e}}}
\end{gathered}
$$

where $q_{\mathrm{e}}$ and $q_{t}\left(\mathrm{mg} \mathrm{g}^{-1}\right)$ are the amounts of $\mathrm{Cd}(\mathrm{II})$ adsorbed at equilibrium and at time $t(\mathrm{~min})$, respectively. $k_{1}\left(\mathrm{~min}^{-1}\right)$ is the rate constant of the pseudo-first-order model and $k_{2}$ is the rate constant of the pseudo-second-order model $\left(\mathrm{g} \mathrm{mg}^{-1} \mathrm{~min}^{-1}\right)$. 


\subsection{Adsorption isotherm}

Isothermal adsorption model is an effective method to evaluate the interaction mechanism and maximum adsorption capacity. $^{26}$ Generally, Langmuir and Freundlich adsorption isothermal models are applied to deal with the situation of water treatment. The Langmuir model is based on the hypothesis that the adsorption process occurs in the form of monolayer adsorption on the homogeneous surfaces, which can be depicted by the following equation:

$$
\frac{C_{\mathrm{e}}}{q_{\mathrm{e}}}=\frac{1}{q_{\mathrm{m}} K_{\mathrm{L}}}+\frac{C_{\mathrm{e}}}{q_{\mathrm{m}}}
$$

Freundlich model normally represents the multilayer adsorption on the heterogeneous solid surfaces, which can be described as:

$$
\ln q_{\mathrm{e}}=\ln K_{\mathrm{F}}+\frac{1}{n} \ln C_{\mathrm{e}}
$$

where $C_{\mathrm{e}}\left(\mathrm{mg} \mathrm{L}^{-1}\right)$ is the final concentration of $\mathrm{Cd}(\mathrm{II})$ in aqueous solutions, and $q_{\mathrm{e}}\left(\mathrm{mg} \mathrm{g}^{-1}\right)$ is the adsorbed amount of Cd(II) on solid phase, and $q_{\mathrm{m}}\left(\mathrm{mg} \mathrm{g}^{-1}\right)$ is the maximum adsorbed amount of Cd(II) on per unit weight of solid. $K_{\mathrm{L}}\left(\mathrm{L} \mathrm{mg}^{-1}\right)$ is Langmuir constant, which is related to the bonding and affinity of $\mathrm{Cd}(\mathrm{II})$ on adsorbents. $K_{\mathrm{F}}\left(\mathrm{mg}^{1-n} \mathrm{~L}^{n} \mathrm{~g}^{-1}\right)$ is Freundlich constant which is related to sorption capacity.

\subsection{Characterization}

The images of synthesized samples before and after adsorbing Cd(II) were captured by a scanning electron microscope (SEM) (JSM-7500F, Japan). The specific surface areas and pore structures of the samples were detected by nitrogen adsorption based on Brunauer-Emmett-Teller (BET) and Barrett-JoynerHalenda $(\mathrm{BJH})$ methods using $\mathrm{N}_{2}$ adsorption-desorption at $77 \mathrm{~K}$ on a surface area analyzer (ASAP2020, USA). The X-ray powder diffraction (XRD) patterns were carried out by a powder diffractometer using $\mathrm{Cu} \mathrm{K} \alpha$ radiation at a scanning speed of $2^{\circ} \min ^{-1}$ (PANalytical B.V., Holland). X-ray photoelectron spectroscopy (XPS) tests were measured on an AXIS Ultra DLD (Shimadzu, Japan) using monochromatic Al Ka X-ray source.

\section{Results and discussion}

\subsection{Structure and morphologies of as-prepared particles}

The structures of the obtained particles were characterized by XRD to determine the crystal structure and its integrity in Fig. S1. $\dagger$ The as-prepared LDH-GO particles were principally composed of a hexagonal LDHs phase and exhibited their characteristic diffractions, such as the peaks [003], [006], [012], [110] and [113], with a $d$-spacing of $0.780 \mathrm{~nm} .{ }^{27}$ It has to be noted that no diffraction peaks of impurities were discerned, which suggested high purity of the sample. The hydrolyzed LDH-GO (H-LDH-GO) exhibited an XRD pattern similar to the pristine $\mathrm{LDH}-\mathrm{GO}$, indicating the good stability of the LDH-GO in the water. The LDH-GO@Fe(II) $x$ particles (LDH-GO@Fe(II) $)_{10}$ and $\mathrm{LDH}-\mathrm{GO}$ (Fe(II) $)_{50}$ ) showed similar diffraction peaks as
LDH-GO particles but slight change in $d$-spacings and relative intensities of peaks. This observation matched well with the structure of LDH-GO, which suggested that the layered structures well retained after Fe(II)-decoration. Compared with the pristine $\mathrm{LDH}-\mathrm{GO}$, the diffraction peaks intensity of the $\mathrm{H}-\mathrm{LDH}-$ GO and LDH-GO@Fe(II) $)_{x}$ were lower, which was due to slightly decomposition of the LDH-GO. The change in crystal lattice parameters in XRD patterns after Fe-modification was due to isomorphic substitution of metal ions in the structure. Isomorphic substitution would lead to the change in the $d$ spacings and relative intensities of peaks that reflected lattice parameters of layer framework. ${ }^{28}$ This might due to partial isomorphic substitution of $\mathrm{Mg}$ (II) by $\mathrm{Fe}(\mathrm{II})$ during the modification. And then the $\mathrm{Fe}(\mathrm{II})$ were oxidized to $\mathrm{Fe}(\mathrm{III})$, which was confirmed by XPS.

Due to the highly variable chemistry of iron, there are various possible structures of Fe species in composites. In most cases, single di- and tri-valent Fe, oxo- and hydroxo-complexes, polymeric oxidic species and iron oxide species are present simultaneously. ${ }^{21}$ The XRD patterns revealed no notable changes in the parent material diffraction peaks, which indicated no detectable damage to the $\mathrm{LDH} / \mathrm{GO}$, nor the presence of additional Fe oxidic or oxo-hydroxidic crystalline phases. ${ }^{21}$ This absence of additional peaks was due to the amorphous nature of the Fe-phases formed at LDH/GO surface sites, which was in line with the previous literatures. ${ }^{20,29}$

The wide scan XPS spectrum of $\mathrm{H}-\mathrm{LDH}-\mathrm{GO}$ and $\mathrm{LDH}-$ GO@Fe(II) $)_{10}$ composites are shown in Fig. 1(a). The most significant features in these spectrums were the $\mathrm{Mg} 1 \mathrm{~s}, \mathrm{Al} 2 \mathrm{p}, \mathrm{Fe}$ $2 \mathrm{p}, \mathrm{O} 1 \mathrm{~s}$, and $\mathrm{C} 1 \mathrm{~s}$ signals. The peaks due to Fe revealed the presence of Fe species in the LDH-GO@Fe(II) 10 $_{10}$ composite. The detailed spectra of the Fe $2 \mathrm{p}$ is illustrated in Fig. 1(b). The peaks observed at $\sim 725$ and $\sim 711 \mathrm{eV}$ were assigned to Fe $2 \mathrm{p}_{1 / 2}$ and Fe $2 \mathrm{p}_{3 / 2}$, respectively. ${ }^{30}$ For stoichiometry evaluation of Fe, only Fe $2 \mathrm{p}_{3 / 2}$ was analyzed, and the Fe $2 \mathrm{p}_{1 / 2}$ was used as reference for the fitting procedure based on previous literatures. ${ }^{31,32}$ The signal of Fe(II) peak contribution was found at about $711.0 \mathrm{eV}$. Another peak observed at about the binding energy of $713.0 \mathrm{eV}$ was assigned to $\mathrm{Fe}(\mathrm{III}) .{ }^{33}$ It revealed the oxidation of $\mathrm{Fe}(\mathrm{II})$ in the structure. The broad $\mathrm{O} 1 \mathrm{~s}$ peaks suggested the existence of various oxygen-containing compounds, referred to organic oxygen ( $\mathrm{O}$ contained in carboxyl, carbonyl and alkoxy groups) and inorganic oxygen. ${ }^{34}$ In Fig. 1(c), three peaks at 529, 530, and $531 \mathrm{eV}$ were attributed to $\mathrm{M}-\mathrm{O}, \mathrm{C}-\mathrm{O} / \mathrm{C}-\mathrm{OH}$, and $\mathrm{H}_{2} \mathrm{O}$ bonds, respectively. ${ }^{35}$

The SEM micrograph and EDS spectra of the H-LDH-GO, LDH-GO@Fe(II) $)_{10}$ and LDH-GO@Fe(II) $)_{50}$ particles are shown in Fig. S2. $\uparrow$ The EDS spectra results revealed that the composition of the $\mathrm{H}-\mathrm{LDH}-\mathrm{GO}$ adsorbent was predominated by $\mathrm{Mg}, \mathrm{Al}, \mathrm{C}$ and $\mathrm{O}$. After modification with $\mathrm{Fe}(\mathrm{II})$, the Fe peak was clearly detected in the LDH-GO@Fe(II) 10 and $\mathrm{LDH}-\mathrm{GO} @ \mathrm{Fe}(\mathrm{II})_{50}$ samples. The results indicated that Fe was successfully grafted onto the LDH-GO surface during the modification process. The surface content of $\mathrm{Fe}$ for $\mathrm{LDH}-\mathrm{GO} @ \mathrm{Fe}(\mathrm{II})_{50}$ was $34.95 \%$ (atomic\%), which was about 5 times than that of LDH$\mathrm{GO} @ \mathrm{Fe}(\mathrm{II})_{10}$. The formation of Fe-decorated microsphere was mainly through the following reaction: (i) the Fe(II) isomorphic 

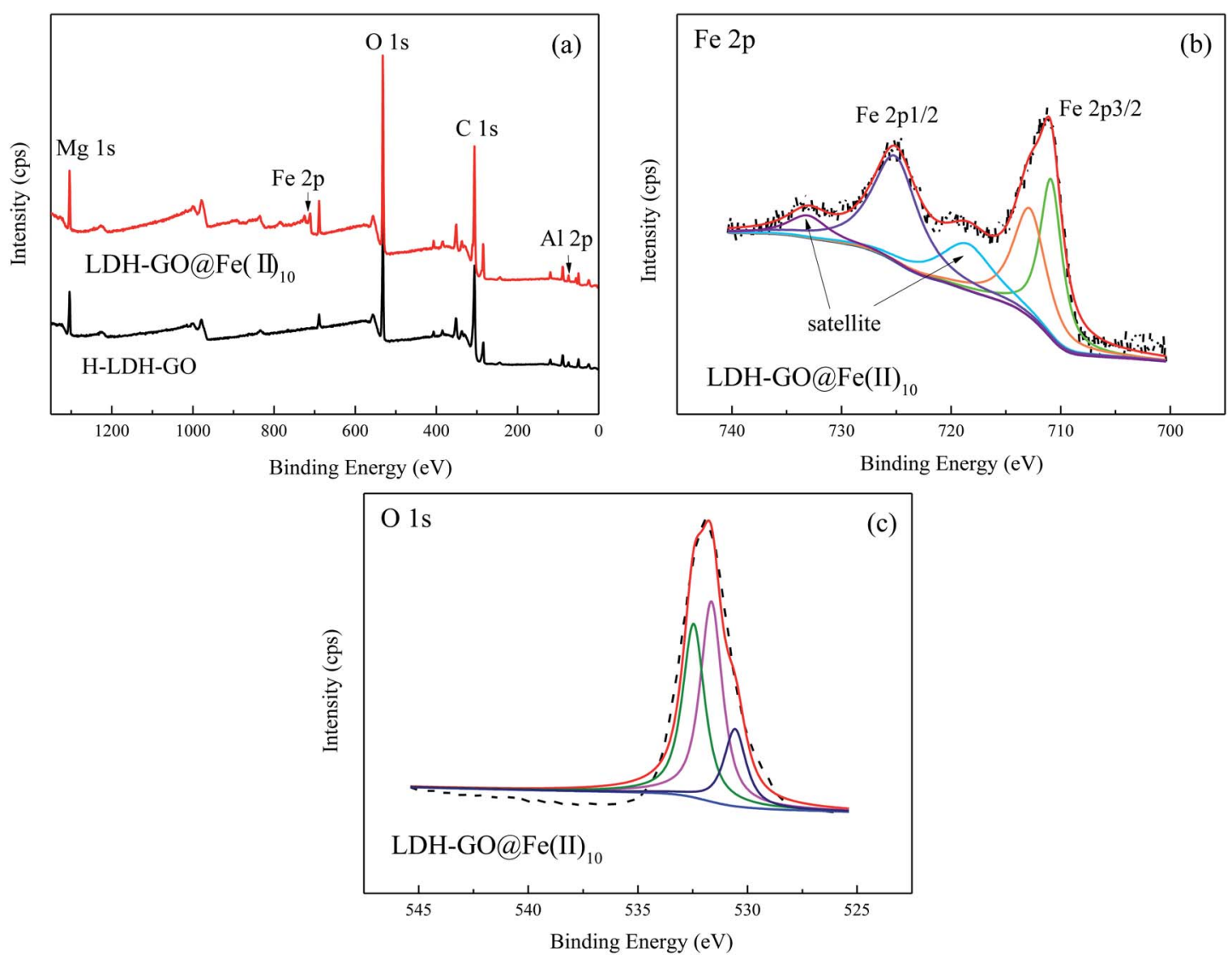

Fig. 1 (a) Wide scan, (b) Fe 2p and (c) O 1s XPS spectra.

substituted of $\mathrm{Mg}$ (II); (ii) partial divalent iron was converted to trivalent iron upon reaction with oxygen (from dissolved air); (iii) with the amount of Fe(III) in the structure increased, the stability of LDH-GO became weaker and a fraction of the structure collapsed and $\mathrm{Fe}(\mathrm{III})$ released into the solution. The released $\mathrm{Fe}(\mathrm{III})$ reacted with $\mathrm{OH}^{-}$forming precipitated $\mathrm{Fe}(\mathrm{OH})_{3}$ that subsequently transformed into $\mathrm{FeO}_{x}{ }^{36}$ (iv) The GO and iron formed strong surface complexes through the Lewis acid-base interaction. As can be seen from Fig. 3(a), the morphologies of H-LDH-GO particles were similar to the LDH-GO reported in other reports. ${ }^{13}$ The LDH sheets arrays grew on both sides of the GO sheets. The inhomogeneous flakes were smooth and flat. The flakes stacked together to form agglomerates in some areas. When the initial concentration of $\mathrm{Fe}(\mathrm{II})$ was $10 \mathrm{mg} \mathrm{\textrm {L } ^ { - 1 }}$, the surface of LDH-GO@Fe(II) ${ }_{10}$ presented a large area continuous porous-flocculent structure, but they did not form separate flakes. When the initial concentration of $\mathrm{Fe}(\mathrm{II})$ increased to $50 \mathrm{mg} \mathrm{L}^{-1}$, the flakes formed the basic substrate and almost every flake was upright, and some flakes clustered together on the top of the substrate. ${ }^{37}$ This may be due to the Fe(II) transferred into parallel lamellae and stretched the distance between the flakes. Moreover, the surface functional groups of the LDHGO possibly formed intensive electrostatic and chemical interactions with metal ions. The interactions could drag Fe(II) into inner space, which lead parallel flakes more loosely and become upright orientation. The results showed that the LDH-
GO@Fe(II) particles had porous structures, which provided effective adsorption sites.

The $\mathrm{N}_{2}$ adsorption and desorption isotherms are used to measure the surface area and pore structure of the obtained particles. The BET and BJH methods are applied to determine the surface area and the pore size distribution considering the desorption and adsorption branch of the $\mathrm{N}_{2}$ isotherms, respectively. The nitrogen adsorption-desorption isotherms are showed in Fig. S3.† All the isotherms were type III-like adsorption isotherms with a H3-type hysteresis loop for the desorption isotherms. The results revealed the particles exhibited mesoporous structures. The obtained specific surface areas were $73.3,84.4$ and $88.3 \mathrm{~m}^{2} \mathrm{~g}^{-1}$ for the H-LDH-GO, LDH-GO@Fe(II) $)_{10}$ and LDH-GO@Fe(II) $)_{50}$, respectively. The average pore sizes of the H-LDH-GO, LDH-GO@Fe(II) $)_{10}$ and LDH-GO@Fe(II) $)_{50}$ were $13.11,11.76$ and $11.73 \mathrm{~nm}$. The pore volume of the H-LDH-GO, LDH-GO@Fe(II) $)_{10}$ and LDH-GO@Fe(II) $)_{50}$ were $0.23,0.24$ and $0.26 \mathrm{~cm}^{3} \mathrm{~g}^{-1}$, respectively. The modification lead to a change in specific surface area and pore size while no significant difference in pore specific volume. The modified LDH-GO (LDH$\mathrm{GO} @ \mathrm{Fe}(\mathrm{II})_{10}$ and $\left.\mathrm{LDH}-\mathrm{GO} @ \mathrm{Fe}(\mathrm{II})_{50}\right)$ had specific surface areas larger than that of the unmodified LDH-GO and thus, and its average pore sizes were smaller. A change in the average pore size range was likely due to the iron formations coating. The iron formations possibly blocked certain pores of the LDH-GO support, in particular small diameter pores. ${ }^{38}$ The results 
indicated that $\mathrm{Fe}$ (II)-decoration affected the pore structure of the LDH-GO, which increased its specific surface area. The increase in specific surface area was due to the presence of noncrystalline Fe formations located at the surface of the LDH/GO crystal. Generally, a poorly crystalline phase is desirable for adsorption because the lack of a 3D crystalline structure results in high specific surface area. ${ }^{21}$ The enlarged surface area and pore size of LDH-GO@Fe(II) $)_{10}$ and LDH-GO@Fe(II) $)_{50}$ particles, made Cd(II) more freely diffuse and moved into the larger mesochannels, and readily be removed.

\subsection{Cd(II) removal by LDH-GO@Fe(II) composites}

It is shown in Fig. 2 that the $\mathrm{Cd}(\mathrm{II})$ adsorption performance depended on the initial $\mathrm{Fe}(\mathrm{II})$ concentrations. The removal rate of $\mathrm{Cd}$ (II) increased from $66.8 \%$ of the $\mathrm{H}-\mathrm{LDH}-\mathrm{GO}$ to $77.2 \%$ of the $\mathrm{LDH}-\mathrm{GO} @ \mathrm{Fe}(\mathrm{II})_{10}$. It is interesting to observe that with the further increase of Fe content in the modified LDH-GO, the removal capacity for $\mathrm{Cd}(\mathrm{II})$ removal had a decline. The $\mathrm{LDH}-$ $\mathrm{GO} @ \mathrm{Fe}(\mathrm{II})_{10}$ composites showed the highest removal capacity for Cd(II).

Generally speaking, adsorbents own large surface area and porosity have great adsorption capacity for adsorbates. The LDH-GO@Fe(II) 10 $_{0}$ composites had smaller surface area and pore volume than LDH-GO@Fe(II) $)_{50}$, but showed higher removal capacities for $\mathrm{Cd}(\mathrm{II})$. This phenomenon demonstrated that doping a certain amount of $\mathrm{Fe}(\mathrm{II})$ into $\mathrm{LDH}-\mathrm{GO}$ particles can enlarge the surface area and provide more adsorption sites. But, excessive Fe(II) will restrain the adsorption for Cd(II). When the composites exposed to Fe(II)-containing solution, isomorphic substitution of $\mathrm{Mg}$ (II) took place. Replacement ions must have the same total ionic charge and approximately the same size as those replace. ${ }^{39} \mathrm{Fe}(\mathrm{II})$ substituted part of $\mathrm{Mg}$ (II) through isomorphous, and then $\mathrm{Fe}(\mathrm{II})$ were oxidized to $\mathrm{Fe}(\mathrm{III})$ on the surface of the LDH-GO. The results of XPS spectrums also confirmed the existence of Fe(III) in the composites. The Fe(III) in the structure of LDH-GO made the brucite-like sheets more positively charged, thus formed an electrostatic repulsion with positively charged $\mathrm{Cd}(\mathrm{II})$. On the other hand, the delocalized $\pi$

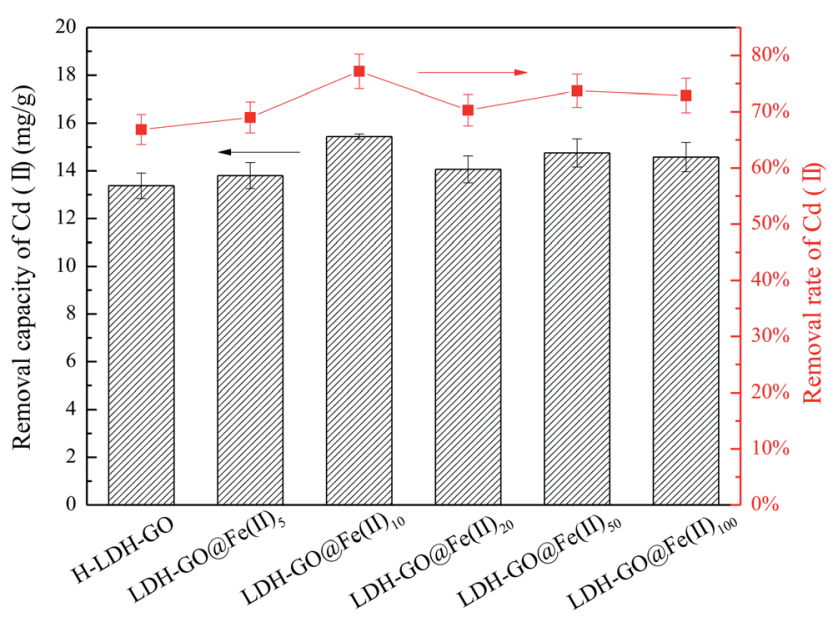

Fig. 2 The removal performance of $\mathrm{Cd}(\|)$ on $\mathrm{LDH}-\mathrm{GO}\left(\mathrm{aFe}(\|)_{x}\right.$. electron systems of graphene layer can act as Lewis base to form electron donor-acceptor complexes with metal ions. ${ }^{40}$ During the decoration of $\mathrm{Fe}(\mathrm{II})$ on the LDH-GO, strong surface complexation between the GO and Fe(II) and Fe(III) occurs through the Lewis acid-base interaction, which also occupied part of the adsorption sites on GO sheets. As a result, $10 \mathrm{mg} \mathrm{L}^{-1}$ was the optimum precursor concentration for LDH-GO modification by $\mathrm{Fe}(\mathrm{II})$.

Adsorption kinetic tests for Cd(II) (Fig. 3) were performed to evaluate the contact time needed for sorption equilibrium, with an initial Cd(II) concentration of $20 \mathrm{mg} \mathrm{L}^{-1}$, the sorbent dosage of $1.0 \mathrm{~g} \mathrm{~L}^{-1}$, the $\mathrm{pH}$ value of 5.0 and the temperature of $25^{\circ} \mathrm{C}$. The equilibrium solution $\mathrm{pH}$ after sorption was about 6 . It is possible that the increase in $\mathrm{pH}$ was due to the alkalinity of the adsorbent. The results are shown in Fig. 3, the adsorption amount significantly increased within the first $4 \mathrm{~h}$, followed by a slow increase until equilibrium was reached. The necessary time to reach equilibrium was about $12 \mathrm{~h}$. To ensure adsorption equilibrium, a contact time of $24 \mathrm{~h}$ was chosen.

The suitable results obtained from different models are summarized in Table 1 . It can be seen that the correlation coefficient of the pseudo-second-order model $\left(R^{2}=0.999\right)$ was much higher than the pseudo-first order. Therefore, the adsorption kinetics followed the pseudo-second-order model, suggesting a chemisorption process. The chemisorption mechanism involved formation of chemical bonds between the adsorbents function groups and the metal ions. ${ }^{\mathbf{4 1 , 4 2}}$

The adsorption isotherm is shown in Fig. 4 and the relative parameters calculated from the two models are listed in Table 2. Based on the Langmuir parameters, LDH-GO@Fe(II) $)_{10}$ exhibited a higher affinity for $\mathrm{Cd}(\mathrm{II})$ than $\mathrm{H}-\mathrm{LDH}-\mathrm{GO}$ and $\mathrm{LDH}-$ $\mathrm{GO} @ \mathrm{Fe}(\mathrm{II})_{50}$, as reflected by its higher adsorption capacity. However, the Fe content of LDH-GO@Fe(II) 10 $_{10}$ was much lower than that of LDH-GO@Fe(II) , $_{50}$, which indicated that the Fe species in LDH-GO@Fe(II) $)_{10}$ were more efficient. The sorption isotherms were better fitted by the Langmuir model than by the Freundlich model. The increased sorption capacities can be

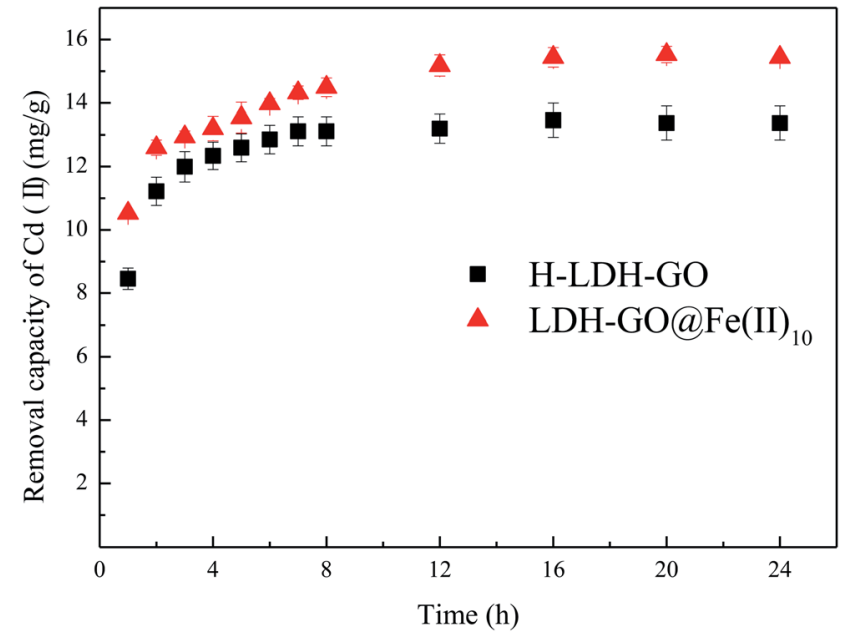

Fig. 3 Effect of contact time on the adsorption of $\mathrm{Cd}(॥)$ onto $\mathrm{H}-\mathrm{LDH}-$ $\mathrm{GO}$ and LDH-GO@Fe(॥) 10 . 
Table 1 Kinetics parameters for $\mathrm{Cd}(॥)$ adsorption on $\mathrm{H}-\mathrm{LDH}-\mathrm{GO}$ and LDH-GOQFe( $(I)_{10}$

\begin{tabular}{|c|c|c|c|c|c|c|c|}
\hline Adsorbents & $q_{\mathrm{e}, \exp }\left(\mathrm{mg} \mathrm{g}^{-1}\right)$ & \multicolumn{3}{|c|}{ Pseudo-first order } & \multicolumn{3}{|c|}{ Pseudo-second order } \\
\hline LDH-GO@Fe(II) $)_{10}$ & 15.43 & 14.69 & 1.2357 & 0.8995 & 15.97 & 0.0871 & 0.9996 \\
\hline
\end{tabular}

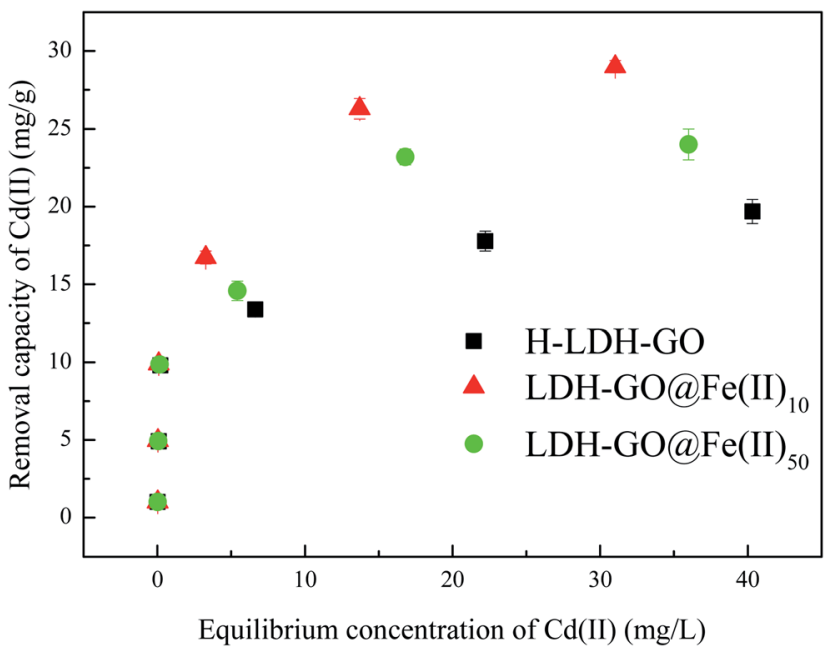

Fig. 4 Adsorption isotherm of $\mathrm{Cd}(\Perp)$ onto $\mathrm{H}-\mathrm{LDH}-\mathrm{GO}, \mathrm{LDH}-$ $\mathrm{GO} @ \mathrm{Fe}\left({ }_{11}\right)_{10}$ and LDH-GO@Fe(॥) 50 .

explained by the increased specific surface area and the high affinity of amorphous Fe oxides with $\mathrm{Cd}(\mathrm{II})$ on the surface of $\mathrm{LDH}-\mathrm{GO} @ \mathrm{Fe}(\mathrm{II})_{10}$. The previous study inferred that the $\mathrm{FeMnO}_{x}$ phases of FMBC contributed to the sorption of $\mathrm{Cd}(\mathrm{II})$ and $\mathrm{Cu}(\mathrm{II})$, the increased uptake was mainly due to the formation of strong mono- or multidentate inner-sphere complexes (e.g., $\mathrm{COO}-\mathrm{M}$ $(\mathrm{M}=\mathrm{Cu}$ or $\mathrm{Cd})$ and $\mathrm{Fe}-\mathrm{Mn}-\mathrm{O}-\mathrm{M}){ }^{34}$ Previous studies showed the oxygenic functional groups on GO can bind with heavy metal ions and form complexes. ${ }^{43,44}$ It can be speculated that the adsorption of $\mathrm{Cd}(\mathrm{II})$ was own to the formation complexes between graphene oxide (e.g., $\mathrm{COO}-\mathrm{Cd}$ and $\mathrm{O}-\mathrm{Cd})$ and $\mathrm{Fe}$ species $\left(\mathrm{FeO}_{x}-\mathrm{Cd}\right)$. Excess $\mathrm{Fe}$ species in $\mathrm{LDH}-\mathrm{GO} @ \mathrm{Fe}(\mathrm{II})_{50}$ occupied the binding sites for $\mathrm{Cd}(\mathrm{II})$ and $\mathrm{Fe}(\mathrm{III})$ in $\mathrm{LDH}-$ GO@Fe(II) $)_{50}$ brought positive charge to adsorbent which hindered $\mathrm{Cd}(\mathrm{II})$ adsorption via electrostatic repulsion.

\subsection{Removal mechanism}

To further investigate the Cd(II) removal mechanisms on the Fedecorated LDH-GO particles, the samples after adsorption of Cd(II) were characterized by XRD, SEM/EDS, and XPS techniques.

Fig. 5(a) illustrates the XRD patterns of the final solid sample after treating $20 \mathrm{mg} \mathrm{L}^{-1} \mathrm{Cd}(\mathrm{II})$. The peaks attributed to the $\mathrm{LDH}-$ $\mathrm{GO} @ \mathrm{Fe}(\mathrm{II})_{10}$ were impaired. The main phases were indexed $\mathrm{Cd}(\mathrm{OH})_{2}$ or/and $\mathrm{Cd}(\mathrm{OH}) \mathrm{Cl},{ }^{45}$ which indicated the precipitation form of cadmium on the surface of LDH-GO@Fe(II) $)_{10}$.

In order to further prove the presence of $\mathrm{Cd}$ on $\mathrm{LDH}_{-}$ $\mathrm{GO} @ \mathrm{Fe}(\mathrm{II})_{10}$, the content of various elements on the surface of LDH-GO@Fe ${ }_{10}$ after Cd(II) adsorption was measured by EDS (Fig. S3†). The EDS spectrum showed $0.80 \%$ (atomic\%) of Cd was loaded on the surface of LDH-GO@Fe(II) $)_{10}$. The content of Fe on the surface of LDH-GO@Fe(II) $)_{10}$ dramatically declined to $0.79 \%$ (atomic\%), compared to the original LDH-GO@Fe(II) ${ }_{10}$ (6.10\%). The content of $\mathrm{O}$ on the surface of LDH-GO@Fe(II) ${ }_{10}$ increased from $46.12 \%$ (atomic\%) to $54.53 \%$ (atomic\%) after $\mathrm{Cd}(\mathrm{II})$ adsorption. This was due to the adsorbed Cd(II) formed Cd hydroxides and Cd-complex with oxygen-containing groups on the surface of LDH-GO@Fe(II) $)_{10}$. The SEM results (Fig. S3 $†$ ) showed that the porous surface was substituted by lumpy structure. The Cd hydroxides cover part of the original porous structure. This study inferred that the LDH-GO@Fe(II) contributed to the sorption of Cd(II) through surface complexation as well as precipitation.

The XPS survey scan of the H-LDH-GO and LDHGO@Fe(II) $)_{10}$ after cadmium adsorption is shown in Fig. 5(b). The result displayed the remarkable presentation of $\mathrm{Cd} 3 \mathrm{~d}$ spectra after cadmium adsorption, which signified the presence of $\mathrm{Cd}$ on the surface of the adsorbents. In Fig. S4(a), $\dagger$ the doublet characteristics of Cd appeared at 406 and $412 \mathrm{eV}$ were in line with those of $\mathrm{Cd}(\mathrm{OH})_{2}$, which indicated the precipitation form of cadmium..$^{20,46}$ Combined with XRD pattern, it is clear

Table 2 Isotherm parameters for $\mathrm{Cd}(॥)$ adsorption on the synthesized materials

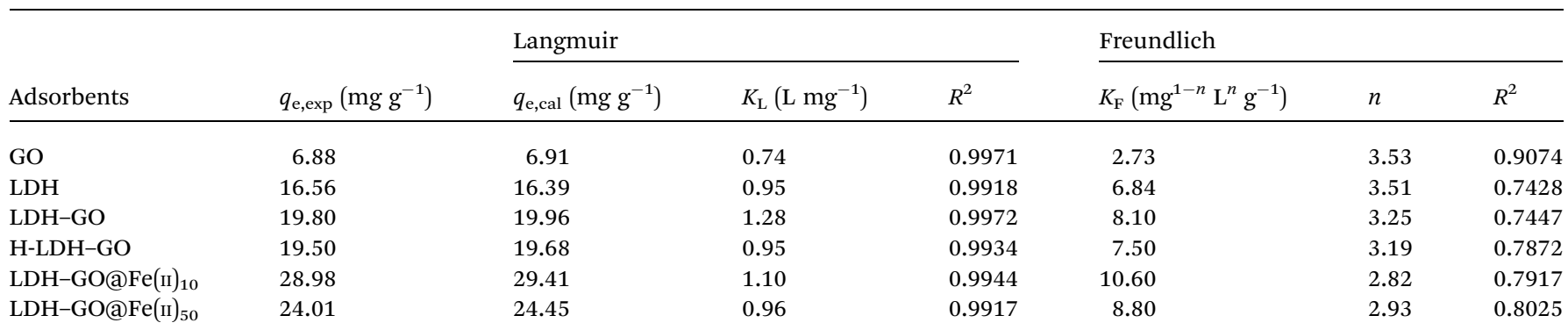



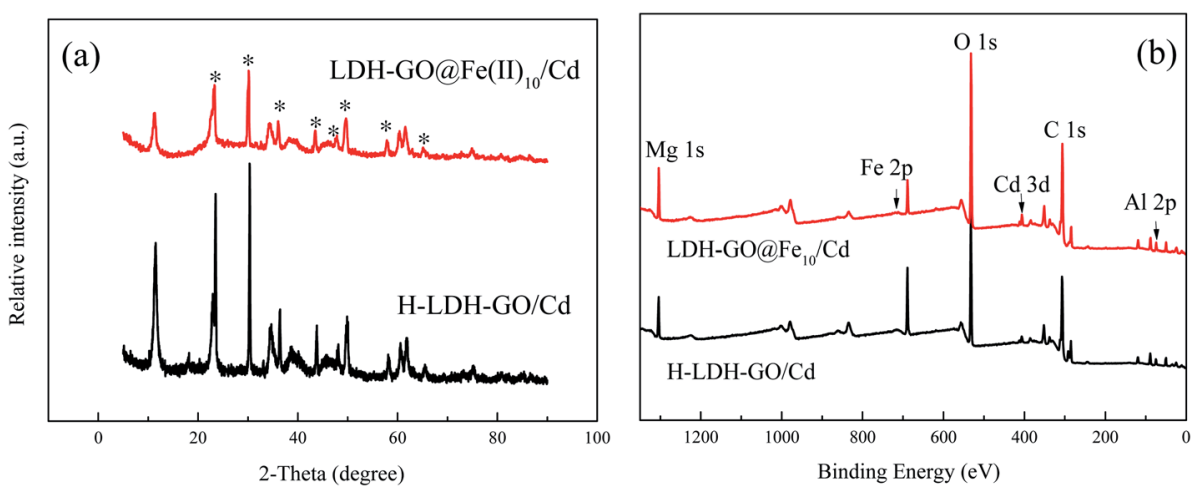

Fig. 5 (a) The XRD patterns of $\mathrm{H}-\mathrm{LDH}-\mathrm{GO}$ and $\mathrm{LDH}-\mathrm{GO}\left(\mathrm{FFe}(\Perp)_{10}\right.$ after adsorption of $\mathrm{Cd}(\Perp)$ (the asterisk signs indicate $\mathrm{Cd}(\mathrm{OH})_{2}$ or $\left.\mathrm{Cd}(\mathrm{OH}) \mathrm{Cl}\right) ;(\mathrm{b})$ XPS survey scan of $\mathrm{H}-\mathrm{LDH}-\mathrm{GO}$ and $\mathrm{LDH}-\mathrm{GO}\left(\mathrm{Fe}\left(\|_{10}\right)_{10}\right.$ after removal of $\mathrm{Cd}(\|)$.

that in this study, the removal of Cd(II) from aqueous solution was mainly conducted by surface-induced precipitation as hydroxides. Notably, the peaks of Fe $2 p$ were impaired after $\mathrm{Cd}(\mathrm{II})$ adsorption (Fig. S4(b)†), which indicated the $\mathrm{Cd}(\mathrm{II})$ hydroxides covered the surface of $\mathrm{LDH}-\mathrm{GO} @ \mathrm{Fe}(\mathrm{II})_{10}$ and $\mathrm{Fe}$ atoms were wrapped in the inner structure.

Combined with the XRD and XPS results, the deposition of $\mathrm{Cd}(\mathrm{II})$ played a significant role in the adsorption process. Complexation between $\mathrm{Cd}(\mathrm{II})$ and $\mathrm{FeO}_{x}$ as well as O-containing groups should also be considered. The $\mathrm{O}$ content on the surface of the adsorbents increased after $\mathrm{Cd}(\mathrm{II})$ adsorption, which might be due to the formation of complex with oxygen-containing groups (such as $\mathrm{COO}-\mathrm{Cd}, \mathrm{O}-\mathrm{Cd}, \mathrm{FeO}_{x}-\mathrm{Cd}$ ). Su et al. ${ }^{47}$ demonstrated that heavy metal sorption on amorphous hydrous manganese dioxide usually related to the inner-sphere complex formation. Thus, complex formation can be included as another contributor for Cd(II) adsorption on the Fe-decorated LDH-GO. Cd(II) removal by the LDH-GO@Fe(II) composites were controlled by chemical adsorption via isomorphic surface-induced precipitation and complexes formation, which was constant with results of pseudo-second-order model. The decoration of Fe(II) on LDHGO facilitated the $\mathrm{Cd}(\mathrm{II})$ adsorption process. With the modification of $\mathrm{Fe}(\mathrm{II})$, non-crystalline Fe formations located at the surface of the LDH/GO crystal. Iron oxides nanoparticles exhibited favorable sorption to heavy metals in terms of high capacity and selectivity. Uheida et al. ${ }^{48}$ demonstrated $\mathrm{Co}(\mathrm{II})$ was effectively removed by iron oxides nanoparticles, and ion exchange and surface complexation were considered as the possible uptake mechanisms. The LDH-GO@Fe(II) $)_{50}$ particles had higher Fe loading than that of LDH-GO@Fe(II) ${ }_{10}$, but a lower removal capacity for $\mathrm{Cd}(\mathrm{II})$. This revealed that the iron oxides formed on the LDH-GO played a role in the adsorption of $\mathrm{Cd}(\mathrm{II})$, but not a major role. The amorphous Fe-phases formed on $\mathrm{LDH} / \mathrm{GO}$ surface resulted in increasement of specific surface area and pore volume. The porous structure can be attributed to the enhancement for $\mathrm{Cd}(\mathrm{II})$ removal. While, excessive $\mathrm{Fe}(\mathrm{II})$ would restrain the adsorption for $\mathrm{Cd}(\mathrm{II})$. The $\mathrm{Fe}(\mathrm{II})$ in the structure substituted part of $\mathrm{Mg}$ (II), and then $\mathrm{Fe}$ (II) was oxidized to $\mathrm{Fe}(\mathrm{III})$, which made the brucite-like sheets more positively charged. Thus, an electrostatic repulsion formed with positively charged $\mathrm{Cd}(\mathrm{II})$.

\section{Conclusions}

In summary, Fe-decorated LDH-GO composites with high active surface area was successfully synthesized. The loading of Fe on LDH-GO was achieved by contacting the powdered LDHGO with $\mathrm{Fe}(\mathrm{II})$-containing solution for $1 \mathrm{~h}$. The LDH$\mathrm{GO} @ \mathrm{Fe}(\mathrm{II})_{10}$ composites had the highest removal rate for $\mathrm{Cd}(\mathrm{II})$. With further increase of Fe content, electrostatic repulsion and amorphous Fe oxides would block the adsorption for $\mathrm{Cd}(\mathrm{II})$. The results of XPS confirmed Fe(III) in the composites after modification with $\mathrm{Fe}(\mathrm{II})$. The XRD and XPS results revealed $\mathrm{Cd}(\mathrm{OH})_{2}$ formed after the adsorption for $\mathrm{Cd}(\mathrm{II})$. The adsorption followed pseudo-second-order kinetics, indicating chemisorption. The equilibrium data were fitted well with the Langmuir isotherm model. Cd(II) removal by the LDH-GO@Fe(II) composites was controlled by chemical adsorption via isomorphic surfaceinduced precipitation and complexes formation. It was noteworthy that Fe-decorated LDH-GO composites had a favorable adsorption capacity and chemical stability for the adsorption of metal ions.

\section{Conflicts of interest}

There are no conflicts to declare.

\section{Acknowledgements}

This research was supported by the International Scientific and Technological Innovation and Cooperation Project of Sichuan (No. 2019YFH0170).

\section{References}

1 S. Arghavani-Beydokhti, M. Rajabi and A. Asghari, Anal. Methods, 2018, 10, 1305-1314.

2 M. A. González, I. Pavlovic and C. Barriga, Chem. Eng. J., 2015, 269, 221-228.

3 X. Lu, X. Huangfu and J. Ma, J. Hazard. Mater., 2014, 280, 7178.

4 M. K. Uddin, Chem. Eng. J., 2017, 308, 438-462. 
5 E. Gutierrez-Segura, M. Solache-Rios, A. Colin-Cruz and C. Fall, J. Environ. Manage., 2012, 97, 6-13.

6 D. Xu, X. Tan, C. Chen and X. Wang, J. Hazard. Mater., 2008, 154, 407-416.

7 N. Fiol, I. Villaescusa, M. Martínez, N. Miralles, J. Poch and J. Serarols, Sep. Purif. Technol., 2006, 50, 132-140.

8 C. Bertagnolli, S. J. Kleinübing and M. G. C. D. Silva, Appl. Clay Sci., 2011, 53, 73-79.

9 Y. Cao, G. Li and X. Li, Chem. Eng. J., 2016, 292, 207-223.

10 M. Q. Zhao, Q. Zhang, J. Q. Huang and F. Wei, Adv. Funct. Mater., 2012, 22, 675-694.

11 X. Ge, J. Liu, X. Song, G. Wang, H. Zhang, Y. Zhang and H. Zhao, Chem. Eng. J., 2016, 301, 139-148.

12 H. N. Tran, D. T. Nguyen, G. T. Le, F. Tomul, E. C. Lima, S. H. Woo, A. K. Sarmah, H. Q. Nguyen, P. T. Nguyen, D. D. Nguyen, T. V. Nguyen, S. Vigneswaran, D. N. Vo and H. P. Chao, J. Hazard. Mater., 2019, 373, 258-270.

13 V. Gbb, O. A. Oyetade, S. Rana, B. S. Martincigh, S. B. Jonnalagadda and V. O. Nyamori, ACS Appl. Mater. Interfaces, 2017, 9, 17290.

14 L. Tan, Y. Wang, Q. Liu, J. Wang, X. Jing, L. Liu, J. Liu and D. Song, Chem. Eng. J., 2015, 259, 752-760.

15 X. Yuan, Y. Wang, J. Wang, C. Zhou, Q. Tang and X. Rao, Chem. Eng. J., 2013, 221, 204-213.

16 W. Liao, H. Wang, H.-q. Li and P. Yang, Environ. Eng. Sci., 2019, DOI: 10.1089/ees.2019.0179.

17 W. M. Gitari, T. Ngulube, V. Masindi and J. R. Gumbo, Desalin. Water Treat., 2013, 53, 1578-1590.

18 C. Luengo, V. Puccia and M. Avena, J. Hazard. Mater., 2011, 186, 1713-1719.

19 X. Sun, C. Hu and J. Qu, Desalin. Water Treat., 2012, 8, 139145.

20 S. He, Y. Li, L. Weng, J. Wang, J. He, Y. Liu, K. Zhang, Q. Wu, Y. Zhang and Z. Zhang, Sci. Total Environ., 2018, 637-638, 69-78.

21 M. K. Doula, Chemosphere, 2007, 67, 731-740.

22 F. Yang, S. Zhang, Y. Sun, K. Cheng, J. Li and D. C. W. Tsang, Bioresour. Technol., 2018, 265, 490-497.

23 S. H. William Jr and R. E. Offeman, J. Am. Chem. Soc., 1958, 80, 1339.

24 F. Shemirani and B. T. S. Akhavi, Anal. Lett., 2001, 34, 21792188.

25 W. Yao, S. Yu, J. Wang, Y. Zou, S. Lu, Y. Ai, N. S. Alharbi, A. Alsaedi, T. Hayat and X. Wang, Chem. Eng. J., 2017, 307, 476-486.

26 C. Meng, W. Zhikun, L. Qiang, L. Chunling, S. Shuangqing and H. Songqing, J. Hazard. Mater., 2018, 341, 198-206.
27 Y. Zheng, B. Cheng, W. You, J. Yu and W. Ho, J. Hazard. Mater., 2019, 369, 214-225.

28 M. Park, C. L. Choi, Y. J. Seo, S. K. Yeo, J. Choi, S. Komarneni and J. H. Lee, Appl. Clay Sci., 2007, 37, 143-148.

29 F. Zhang, Y. Song, S. Song, R. Zhang and W. Hou, ACS Appl. Mater. Interfaces, 2015, 7, 7251-7263.

30 J. Li, X. Dou, H. Qin, Y. Sun, D. Yin and X. Guan, Water Res., 2019, 148, 70-85.

31 K. Volgmann, F. Voigts and W. Maus-Friedrichs, Surf. Sci., 2012, 606, 858-864.

32 H. Li, J. Wan, Y. Ma, M. Huang, Y. Wang and Y. Chen, Chem. Eng. J., 2014, 250, 137-147.

33 X. Yin, W. Liu and J. Ni, Chem. Eng. J., 2014, 248, 89-97.

34 Q. Zhou, B. Liao, L. Lin, W. Qiu and Z. Song, Sci. Total Environ., 2018, 615, 115-122.

35 M. Liu, Y. Wang, L. Chen, Y. Zhang and Z. Lin, ACS Appl. Mater. Interfaces, 2015, 7, 7961-7969.

36 X. He, X. Qiu and J. Chen, Colloids Surf., A, 2017, 516, 362374.

37 Y. Zhou, X. Li, K. Wang, F. Hu, C. Zhai, X. Wang and J. Bai, J. Alloys Compd., 2019, 770, 6-16.

38 F. Unob, B. Wongsiri, N. Phaeon, M. Puanngam and J. Shiowatana, J. Hazard. Mater., 2007, 142, 455-462.

39 X. Liang, Y. Zang, Y. Xu, X. Tan, W. Hou, L. Wang and Y. Sun, Colloids Surf., A, 2013, 433, 122-131.

40 L. Li, L. Fan, M. Sun, H. Qiu, X. Li, H. Duan and C. Luo, Colloids Surf., B, 2013, 107, 76-83.

41 A. A. Bakr, N. A. Sayed, T. M. Salama, I. O. Ali, R. R. Abdel Gayed and N. A. Negm, Egypt. J. Pet., 2018, 27(4), 1215-1220.

42 M. A. Badawi, N. A. Negm, M. T. H. Abou Kana, H. H. Hefni and M. M. Abdel Moneem, Int. J. Biol. Macromol., 2017, 99, 465-476.

43 C. J. Madadrang, H. Y. Kim, G. Gao, N. Wang, J. Zhu, H. Feng, M. Gorring, M. L. Kasner and S. Hou, ACS Appl. Mater. Interfaces, 2012, 4, 1186-1193.

44 L. Fan, C. Luo, M. Sun, X. Li and H. Qiu, Colloids Surf., B, 2013, 103, 523-529.

45 H. Zhou, Z. Jiang, S. Wei and J. Liang, Water, Air, Soil Pollut., 2018, 229, 78.

46 W. Wang, R. He, T. Yang, Y. Hu, N. Zhang and C. Yang, RSC Adv., 2018, 8, 25754-25766.

47 Q. Su, B. Pan, S. Wan, W. Zhang and L. Lv, J. Colloid Interface Sci., 2010, 349, 607-612.

48 A. Uheida, G. Salazar-Alvarez, E. Bjorkman, Z. Yu and M. Muhammed, J. Colloid Interface Sci., 2006, 298, 501-507. 